\title{
SOME REMARKS ON SYMMETRIC AND FROBENIUS ALGEBRAS
}

\author{
J. P. JANS
}

In [5] we defined the concepts of Frobenius and symmetric algebra for algebras of infinite vector space dimension over a field. It was shown there that with the introduction of a topology and the judicious use of the terms continuous and closed, many of the classical theorems of Nakayama $[7,8]$ on Frobenius and symmetric algebras could be generalized to the infinite dimensional case. In this paper we shall be concerned with showing certain algebras are (or are not) Frobenius or symmetric. In Section 3, we shall see that an algebra can be symmetric or Frobenius in "many ways". This is a problem which did not arise in the finite dimensional case.

In Section 1, we consider algebras of transformations in an infinite dimensional vector space. We show that the algebras of transformations of finite rank are symmetric, but that the algebra of all transformations is not even Frobenius. The latter statement is proved by means of a lemma which shows (among other things) that every transformation in an infinite dimensional vector space is actually a commutator.

In Section 2 we consider tensor products of Frobenius and symmetric algebras. We also show that under certain conditions the inner product on a Frobenius algebra can be normalized so that $(1,1)=1$.

In Section 3, we show that the polynomial ring with coefficients in a field can be made into a symmetric algebra in an uncountable number of ways. As a consequence of this, the polynomial ring has an uncountable number of inequivalent (metrizable) topologies such that it is a topological ring with respect to each and in every such topology every non zero ideal is dense in the whole algebra.

The term topological ring here requires, along with the usual assumptions about addition, only that multiplication by each single element be a continuous

Received September 9, 1959. 
function of the ring into itself. In [5] we gave an example of a symmetric algebra which was a topolocal ring in this sense, but in which multiplication was not continuous on the product of the ring with itself.

1. Algebras of transformations. The classical theory of Frobenius algebras as developed by Nakayama in $[7,8]$ shows that the algebra of all transformations in a finite dimensional vector space is symmetric. In this section we study algebras of transformations in an infinite dimensional space. The main results of this section show that the algebras of continuous transformations of finite rank are symmetric, but that the algebra of all transformations is not even Frobenius.

If $V$ is a vector space over a field $L$ and $V^{\prime}$ a subspace of $\operatorname{Hom}_{L}(V, L)$ which distinguishes points of $V$ then $V^{\prime}$ induces a topology on $V$, see [2, Chapter IV]. It is convenient to use the bracket notation; $\left(x, x^{\prime}\right)$ is the image in $L$ of $x \in V$ under $x^{\prime} \in V^{\prime}$. Following [2] we let $R\left(V, V^{\prime}\right)$ be the algebra of all continuous transformations of finite rank in $V$. Let $R$ be the algebra generated by $R\left(V, V^{\prime}\right)$ and the identity transformation.

THEOREM 1.1. $R$ is a symmetric algebra.

Proof. We first write $R=L+R\left(V, V^{\prime}\right)$, a vector space direct sum where $L$ represents all field multiples of the identity. In the following we define a linear functional on $R$. We first define $\psi^{\prime}(1)=0$. Now, if $T \in R\left(V, V^{\prime}\right)$ then for $x$ in $V, x T$ has the form

$$
x T=\sum_{1}^{n}\left(x, y_{i}^{\prime}\right) y_{i}
$$

see $\left[2\right.$, Chapter IV] for details. We can now define $\psi(T)=\sum_{1}^{n}\left(y_{i}, y_{i}^{\prime}\right)$.

We note that this gives a linear functional on $R$. We define the inner product on $R$ by the equation $(T, S)=\psi(T S)$.

We must show that $\psi(S T)=\psi(T S)$. Also if $T \neq 0$ we must show that there exists $S$ such that $\psi(T S) \neq 0$. It is sufficient to consider finite rank transformations. Let $x T=\sum_{1}^{n}\left(x, y_{i}^{\prime}\right) y_{i}$, then $x T S=\sum_{1}^{n}\left(x, y_{1}^{\prime}\right) y_{i} S$ and $\psi(T S)=\sum_{1}^{n}\left(y_{i} S, y_{i}^{\prime}\right)$. Then $x S T$ can be written $\sum_{1}^{n}\left(x S, y_{1}^{\prime}\right) y_{i}=\sum_{1}^{n}\left(x, S^{\prime} y_{i}^{\prime}\right) y_{i}$, where $S^{\prime}$ is the adjoint transformation of $S$. Thus, $\psi^{\prime}(S T)=\sum_{1}^{n}\left(y_{i}, S^{\prime} y_{i}^{\prime}\right)$. But the equation $\left(y_{i}, S^{\prime} y_{i}^{\prime}\right)$ $=\left(y_{i} S, y_{i}^{\prime}\right)$ holds for all $i$, so $\psi(S T)=\psi(T S)$. 
If $T$, a finite rank transformation, is not zero then a multiple of it is rank 1. Assume that $T$ is rank one then $x T=\left(x, y^{\prime}\right) y$ where $y^{\prime} \neq 0$ and $y \neq 0$. Then there exists $S$ of finite rank such that $y S$ is not in the kernel of $y^{\prime}$. It follows that $\phi(T S)=\left(y S, y^{\prime}\right) \neq 0$ and the inner product defined by $\psi$ distinguishes points. Thus $R$ is a symmetric algebra with respect to that inner product. We note that the linear functional $\psi$ defined on $R\left(V, V^{\prime}\right)$ is the infinite dimensional analog of the trace function. We also remark that the topology induced on $R$ by the inner product we have defined is comparable to but weaker than the topology $R$ receives as a space of functions on $V$, (the product topology in [2, Chapter II]).

We now consider the algebra of all transformations in $V$. The following lemma will help us prove our theorem.

Lemma 1.2. If $T$ is in the algebra of all linear transformations in a vector space of dimension $\geq \S_{0}$ then any equation of the form $Y f(Z)-Z Y=T$ can be solved for $Y$ and $Z$.

Proof. The idea of the proof is to use $Z$ to push the difficulties out to the "end" of a basis of $V$. Since there isn't any end to the basis, the difficulties are gone. Let $B$ be a basis of $V$. Among the well orderings of $B$ there is one in which $B$ fails to have a last element. Here we are using the assumption that the dimension of $V$ is $\geq \S_{0}$. For each $x_{x} \in B$ define $x_{x} Z=x_{x+1}$ where $\alpha+1$ is the successor of $\alpha$ in the well ordering. If $\alpha$ is a limit ordinal we can define $x_{\alpha} Y$ to be anything in $V$ ( 0 will do). If $\alpha=\beta+1$ we define $x_{x} Y=x_{3} Y f(Z)$ $-x_{\beta} T$. By transfinite induction this defines $x_{\alpha} Z$ and $x_{\alpha} Y$ for all $x_{\alpha}$ in $B$. Since $B$ is a basis of $V, Z$ and $Y$ can be considered as linear transformations in $V$. But for a basis element $x_{\beta}$ in $B$, we have the equation $x_{\beta} T=x_{\beta} Y f(Z)-x_{\beta+1} Y$ $=x_{\beta} Y f(Z)-x_{\beta} Z Y$. Thus, $T=Y f(Z)-Z Y$.

A special case of the above situation occurs when $f(Z)=Z$. In that case, the lemma shows that in the ring of transformations on an infinite dimensional vector space every transformation is a commutator. We note that this does not hold in the case that the space is finite dimensional. In that case, no transformation with a non zero trace can be a commutator. Another use of the lemma occurs in the proof of the following

Theorem 1.3. The algebra of $\Lambda$ all linear transformations on an infinile 
dimensional vector space is not a Frobenius algebra.

Proof. Suppose the contrary, that is, $\mathrm{A}$ is equipped with an inner product $(Y, Z)$ satisfying $(X Y, Z)=(X, Y Z)$ and distinguishing elements of $A$. We suppose also that it has Nakayama's automorphism $\alpha$ such that $\left(Y, Z^{\alpha}\right)=(Z, Y)$ for all $Y$ and $Z$ in A. Since 1 , the identity transformation is not zero there exists $T$ such that $(1, T) \neq 0$. By the above Lemma we can solve the equation $Y Z^{\alpha}-Z Y=T$. But then $(1, T)=\left(1, Y Z^{\alpha}-Z Y\right)=\left(1, Y Z^{\alpha}\right)-(1, Z Y)=\left(Y, Z^{\alpha}\right)$ $-(Z, Y)=0$. This contradiction proves the theorem.

The theorem really proves that algebras in which equations of the form $Y Z^{\alpha}-Z Y=T$ can be solved for all automorphisms $\alpha$ and all $T$ are not Frobenius algebras. P. M. Cohn has studied a class of simple algebras in which the equation $a x-x b=c$ has a solution $x$ for all $a, b \neq 0$ and all $c$ [1]. These algebras do not have identities. However, such an algebra can be embedded in an algebra with identity. A slight variation of the proof of Theorem 3.1 then shows the enlarged algebras is not a Frobenius algebra.

In comparing Theorems 1.1 and 1.3, we could not say how "far up" in the algebra of linear transformations one could go before the algebras stop being Frobenius. We also cannot say when the solutions $Y$ and $Z$ of Lemma 1.2 will be continuous relative to some space $V^{\prime}$ of functionals on $V$. If we could always insure continuous solutions then we could insert "continuous" before "linear transformations" in Theorem 1.3.

2. Tensor products and normalizations. In this section we include several results which should have been in [5]. The finite dimensional formulations of these appeared in $[7,8]$.

TheOREM 2.1. The tensor product of two Frobenius (symmetric) algebras is also Frobenius (symmetric).

Proof. Suppose that $A$ and $B$ are Frobenius (or symmetric) over the field $L$ with respect to the two inner products $(x, y)$ and $\langle r, s\rangle$ respectively. For the generators of $A \otimes{ }_{L} B$ we define $[x \otimes r, y \otimes s]=(x, y)\langle r, s\rangle$. We claim that with respect to this inner product $A \otimes_{L} B$ will be Frobenius (symmetric). The only point which requires verification is that this new inner product separates point in $A \otimes{ }_{L} B$. 
Suppose that $\sum_{1}^{n} x_{i} \otimes s_{i} \neq 0$. We may assume that $x_{1}, \ldots, x_{n}$ are independent and similarly with the $s_{i}$. In $A$ the inner product distinguishes points, consequently it induces every linear functional on the finite dimensional space spanned by the $x^{\prime} s$. That is, there exists $y$ in $A$ such that $\left(x_{1}, y\right)=1$ and $\left(x_{i}, y\right)=0$ for $i=2, \ldots, n$. By a similar argument in $B$ there exists $t$ such that $\left\langle s_{1}, t\right\rangle=1$ and $\left\langle s_{i}, t\right\rangle=0$ for $i=2, \ldots, n$. But then $\left[\sum_{1}^{n} x_{i} \otimes s_{i}, y \otimes t\right]=1$ and the inner product distinguishes points in $A \otimes_{L} B$.

Corollary 2.2. If $A$ is Frobenius (symmetric) over $L$ then so in $A_{n}$ the algebra of $n \times n$ matrices with coefficients in $A$.

Proof. It is enough to note that $A_{n}$ is isomorphic to $A \otimes{ }_{L} L_{n}$ and that $L_{n}$ is symmetric.

We remark that the inner product just defined on $A \otimes B$ will not, in general, induce on $A \otimes 1$ (isomorphic to $A$ ) the original inner product. In fact, if $\langle 1,1\rangle$ $=0$ where $\langle x, y\rangle$ is the inner product on $B$, then the restricted inner product on $A \otimes 1$ will be identically equal to zero.

This brings up the question of whether an algebra $B$, Frobenius with respect to an inner product $\langle x, y\rangle$, is also Frobenius with respect to an inner product $(x, y)$ inducing the same topology in $B$ and such that $(1,1)=1$. If this is the case, we know by Theorem 3.1 of [5] that there exists a unit $g$ of $B$ such that $\langle x, y\rangle=(x, y g)$ for all $x, y$ in $B$. The converse is also true. It is natural to say that the two inner products related in this way are equivalent. This leads us to the following normalization theorem.

THEOREM 2.3. If $B$ is a Frobenius algebra with respect to an inner product $\langle x, y\rangle$ and if $B$ is not Jacobson semisimple then there exists an equivalent inner product $(x, y)$ such that $(1,1)=1$.

Proof. If $\langle 1,1\rangle=a \neq 0$, let $(x, y)=\langle x, y\rangle a^{-1}$ and this will do the job. In the following it will be enough to find an equivalent inner product $(x, y)$ such that $(1,1) \neq 0$.

Let $G$ be the group of units of $B$ and suppose that $\langle 1, g\rangle=0$ for all $g$ in $G$. Then for each $n$ in $N$, the Jacobson radical of $B,\langle 1, n\rangle=\langle 1,1\rangle-\langle 1,1-n\rangle$ $=0$. But we are assuming that there exists $n \neq 0$ in $N$. Then $\langle 1, x n\rangle=\langle x, n\rangle$ $=0$ for all $x$ in $B$ which contradicts the assumption that $B$ was Frobenius. 
Thus there exists $g$ in $G$ such that $\langle 1, g\rangle \neq 0$. Now let $(x, y)=\langle x, y g\rangle$ and note that $(1,1) \neq 0$.

We add here that if $B$ is Jacobson semisimple then the normalization given in the above theorem is not, in general, possible. See the polynomial examples in the next section.

3. Examples. As a consequence of Theorem 3.1 of [5] we know that two inner products on a Frobenius aigebra which induce the same topology are actually equivalent in the sense defined above. In this section we will show by example that an algebra can be Frobenius with respect to lots of inequivalent inner products. This varies from the classical theory $[7,8]$ since there all the topologies are equivalent (all discrete).

Let $L$ be a field and $L[x]$ the polynomial algebra over $L$. We shall make $L[x]$ symmetric in an uncountable number of ways. Choose an increasing sequence of positive integers $n_{i}$ such that $n_{i}-n_{i-1} \geq i$ and then define $\phi\left(x^{n_{i}}\right) \neq 0$, $\phi$ of other powers of $x$ to be zero. Extend $\phi$ to be a linear functional in $L[x]$. Now $\phi$ induces an inner product on $L[x]$ by the equation $\phi(f g)=(f, g)$. One readily verifies that $L[x]$ is symmetric with respect to this inner product. We note, however, that any infinite subsequence of the sequence $n_{i}$ will also define an inner product on $L[x]$ and it will be symmetric with respect to that inner product. No two of these inner products are equivalent, since the unit group of $L[x]$ consists only of nonzero field elements. Thus $L[x]$ has an uncountable number of inequivalent inner products and is symmetric with respect to each of them. Actually, it is not hard to show that the cardinality of the set of inequivalent inner products is the greater of $2^{\text {sio }}$ and the cardinality of $L$.

We remark that the polynomial algebra $L[x]$ is a special case of a free algebra $[3,6]$. It can be shown by a rather complicated computation that certain free algebras without nilpotent one sided jdeals are symmetric. In particular, if $S$ is the direct sum of copies of the field and $M$ is a two sided module over $S$ of finite dimension over the field then the free algebra $F(S, M)$ is symmetric if it has no nilpotent one sided ideals.

Now that we have all these different inner products (and different topologies) on the polynomial algebra it might be of some interest to consider some properties of these topologies. The following theorem is an immediate corollary to Theorem 6.3 of [5]. 
Theorem 3.1. If $A$ is a Frobenius algebra over a field $L$ and if $A$ has no $L$ finite dimensional one sided ideals (nonzero) then every one sided ideal of finite L-index in $A$ is dense in $A$ in the topology induced by the inner product on $A$.

But the polynomial algebras have no non zero finite dimensional ideals and every non zero ideal has finite index. Thus, in the symmetric polynomial algebras defined above, every non zero ideal is dense. This is quite unlike any of the usual topologies on the polynomial ring.

We note that all the topologies put on the polynomial algebra are metrizable.

We also note that a. Frobenius algebra satisfying the hypotheses of Theorem 3.1 is all "big radical". Perhaps the following remarks are in order concerning the big radical. In [5] we defined the "big radical" to be the intersection of the closed maximal right ideals of finite field index in the algebra. It is becoming increasingly clear that this characteristic ideal is a little big to be called a radical. For instance, for the polynomial algebras it is the whole algebra. It has been suggested that the ideal be called "the liberal" instead of the radical. The use of this term is motivated by the following politico-mathematical observation: If all the radicals were factored out there would still be quite a bit of structure left. However, if all the liberals were factored out, the world would be quite dull.

\section{BIBLIOGRAPHY}

[1] Cohn, P. M., On a Class of Simple Rings, Mathematika, vol. 5 (1958), pp. 103-117.

[2] Jacobson, N., Structure of Rings, Am. Math. Soc. Coll. Pub. XXXVII (1956).

[3] Jans, J. P., On Segregated Rings and Algebras, Nagoya Math. Jour., vol. 11 (1957), pp. $1-7$.

[4] Jans, J. P., Compact Rings with Open Radical, Duke Math. Jour. vol. 24 (1957), pp. 573578.

[5] Jans, J. P., On Frobenius Algebras, Annals of Math. vol. 69 (1959), pp. 392-407.

[6] Jans, J. P. and Nakayama, T., On the Dimensional of Modules and Algebras, VII, Nagoya Math. Jour. Vol. 11 (1957), pp. 67-76.

[7] Nakayama, T., On Frobenius Algebras I, Annals of Math. vol. 40 (1939), pp. 611-633.

[8] Nakayama, T., On Frobenius Algebras II, Annals of Math. vol. 42 (1941), pp. 1-21.

University of Washington 